\title{
On the Mean Duration of Random Walks
}

\author{
Wolfgang Wasow
}

\begin{abstract}
The mean duration of a discrete random walk in a bounded domain is studied for general transition probability functions. The mean duration satisfies an integral equation which, for small mean step length, can be approximated by an elliptic differential equation. This leads to explicit inequalities for the mean duration in an important special case.
\end{abstract}

\section{Introduction}

This paper is concerned with random walks in a bounded open domain $G$ in $n$-dimensional Euclidean space. The walk ends when the moving point leaves $G$ for the first time. The nature of the random walk is characterized by the transition distribution function $F(Q, P)$. Here $P$ and $Q$ are points of the space, and $F(Q, P)$ is the probability that a point at $P$ will, after one step, be in the rectangular sector of the space in which all coordinates are less than those of $Q$.

By duration of a random walk we mean the total number of steps of the walk, including the one on which the point leaves $G$. The subject of this paper is the mean duration; that is, the expected value $W(P)$ of the duration as a function of the starting point $P$.

It will be shown that - under very general conditions- $W(P)$ is the unique solution of the integral equation (9). In section III assumptions are introduced, which, in a sense to be specified below, make the occurrence of all but very small single steps very unlikely. It will be shown that under those assumptions $W(P)$ differs very little from the solution of a certain elliptic differential equation. This differential equation involves only the first and second moments of $F(P, Q)$. The method used in these sections is closely related to that of Petrovsky $[4] .^{2}$

For a particularly important class of random walks this differential equation reduces to a special case of Poisson's equation. For such random walks it is possible to obtain numerical inequalities for the mean duration. These estimates confirm the plausible conjecture that the mean duration does not grow very fast with the number of dimensions, a fact that is of interest in connection with the attempts to use stochastic sampling procedures for the numerical solution of partial differential equations.

If the moving point performs a continuous Brownian motion rather than a discrete random walk, the mean duration satisfies exactly a Poisson equation. This is an easy consequence of the fact that the fundamental solution of the differential equation for diffusion in the presence of absorbing boundaries can be interpreted as the probability density that a Brownian particle starting from a

\footnotetext{
1 The preparation of this paper was sponsored (in part) by the Office of Naval

Research.
2 Figures in brackets indicate the literature references at the end of this paper.
}

given point $P_{0}$ at time 0 be at a point $P_{1}$ at time $t$ without having met the boundary. (See, e. g. Smoluchovski, Drei Vorlesungen Über Diffusion, etc., Physikalische Zeitschrift 17, p. 568 to 570 (1916), or P. Levi, Les processus stochastiques, p. 272 to 273 (Paris, 1948)). It has been pointed out to the author by M. Kac that this fact could be used as a starting point for an alternative treatment of our problem based on the theory of probability measures in function space.

In sections I to III, where the $n$-dimensional case differs from the two-dimensional one only by the need for more cumbersome notation, we shall give the arguments for two dimensions only.

\section{Preparations; Lemmas on Integral Equations}

The transition distribution function $F(Q, P)$ is assumed to be bounded and Borel measurable for all $P$ and $Q$ and a distribution function with respect to $Q$. In order to guarantee that the moving point leaves the domain $G$ with probability one we introduce a hypothesis which will be called assumption (A).

Assumption $(A)$. There exist two positive numbers $r$ and $s$, independent of $P$, such that the probability is at least $s$ that the abscissa of the moving point be increased by $r$ or more in one step.

We refer to [4, p. 431] for the simple proof that the probability of leaving $G$ is indeed one, if assumption (A) is satisfied.

Denote by $F_{m}(Q, P), m=1,2, \ldots$, the iterated functions defined by

$$
\begin{aligned}
& F_{1}(Q, P)=F(Q, P) \\
& F_{m}(Q, P)=\int_{G} F_{m-1}(Q, R) d F(R, P) .
\end{aligned}
$$

Here, and in the sequel, the Stieltjes differentials are formed with respect to the first argument, and an integral without explicitly indicated domain of integration is to be extended over the whole space $E$.

$F_{m}(Q, P)$ is the probability of a point being in the domain $x<x_{Q}, y<y_{Q}$ after exactly $m$ steps, starting from $P$ and having all of its intermediate positions in $G$. Here $\left(x_{Q}, y_{Q}\right)$ are the coordinates of $Q$. In consequence of assumption (A), and the boundedness of $G$, there exists a positive integer $m$ and a 
positive number $c$, less than one, such that

$$
\int_{G} d F_{m}(Q, P)<c \text {, for all } P \text { in } G \text {. }
$$

We shall need the following lemma concerning integral equations formed with distribution functions. For a proof, we refer again to [4].

Lemma 1: If assumption (A) is satisfied, there exists exactly one bounded function $u(P)$ which solves the integral equation problem

$$
u(P)=\left\{\begin{array}{l}
\int u(Q) d F(Q, P) \text { in } G \\
\psi(P) \text { outside } G,
\end{array}\right.
$$

where $\psi(P)$ is a prescribed bounded and Borel measurable function. This solution can be found by means of successive iterations starting from any function that is equal to $\psi(P)$ outside $G$, and bounded and Borel measurable in $G$.

Corollary: The solution $u(P)$ of (2) satisfies the inequalities

$$
\text { g.l.b. } \psi(P) \leq u(P) \leq \text { l. u. b. } \psi(b) \text {. }
$$

Proof of the corollary: Take as initial approximation to $u(P)$ the function $u_{0}(P)$, which is equal to g.l.b. $\psi(P)$ in $G$. Then

$$
u_{1}(P)=\int u_{0}(Q) d F(Q, P) \geq \text { g.l.b. } \psi,
$$

and, by induction, $u_{n} \geq$ g.l.b. $\psi$. A passage to the limit proves the first one of the inequalities (3). The proof of the second inequality is analogous.

Lemma 2: Let $\phi(P)$ be nonnegative and Borel measurable in $G$, and assume that assumption (A) is satisfied. Then the integral equation

$$
u(P)=\int_{G} u(Q) d F(Q, P)+\phi(P)
$$

has a unique bounded solution in $G$. If $\phi_{n}(P)$ is a nondecreasing sequence of nonnegative functions converging to $\phi(P)$, and $u_{n}(P)$ is defined by

$$
u_{0}=0, u_{n+-1}(P)=\int_{G} u_{n}(Q) d F(Q, P)+\phi_{n}(P),
$$

then

$$
U(P)=\lim _{n \rightarrow \infty} u_{n}(P)
$$

is the solution of (4). The solution $u(P)$ is nonnegative.

Proof: The sequence $u_{n}(P)$ is proved to be nondecreasing by means of mathematical induction, using the relations

$$
u_{1}(P)-u_{0}(P)=\int_{G} \phi_{1}(Q) d F(Q, P) \geq 0,
$$

and

$$
\begin{aligned}
u_{n+1}(P)-u_{n}(P)= & \int_{G}\left[u_{n}(Q)-u_{n-1}(Q)\right] d F(Q, P)+ \\
& \phi_{n+1}(P)-\phi_{n}(P) .
\end{aligned}
$$

In order to show also the boundedness of the sequence $u_{n}(P)$ we iterate $(15)$ and find

$$
\begin{aligned}
u_{n+1}(P) & =\int_{G} \int_{G} u_{n-1}(Q) d F(Q, R) d F(R, P)+\phi_{n}^{(2)}(P) \\
& =\int_{G} u_{n-1}(Q) d Q\left[\int_{G} F(Q, R) d F(R, P)\right]+\phi_{n}^{(2)}(P) \\
& =\int_{G} u_{n-1}(Q) d F_{2}(Q, P)+\phi_{n}^{(2)}(P)
\end{aligned}
$$

where

$\phi_{n}^{(2)}(P)=\int_{G} \phi_{n-1}(Q) d F(Q, P)+\phi_{n}(P) \leq 21$. u. b. $\phi(P)$.

Repeating this procedure $m$ times-assuming $n \geq m$ - the integral equation

$$
u_{n+1}(P)=\int_{G} u_{n-m}(Q) d F_{m}(Q, P)+\phi_{n}^{(m+\mathbf{1})}(P)
$$

is obtained, where

$$
\phi_{n}^{(m+1)}(P) \leq(m+1) \text { 1. u. b. } \phi(P) .
$$

If $L_{n}$ denotes the $1 . \mathrm{u}$. b. of $u_{n}(P)$ in $G$ we have therefore, using (1),

$$
\begin{aligned}
& L_{n+1} \leq c L_{n-m}+(m+1) 1 \text {. u. b. } \phi(P) \\
& \quad \leq c L_{n}+(m+1) \text { 1. u. b. } \phi(P),
\end{aligned}
$$

hence

$$
L_{n} \leq(1-c)^{-1} m \text { 1. u. b. } \phi(P) \text {. }
$$

The sequence $u_{n}(\mathrm{P})$, being bounded and nondecreasing has therefore a limit, and this limit satisfies the integral equation (4), since a passage to the limit under the integral sign in (5) is legitimate.

The uniqueness of the solution follows by a standard argument from the uniqueness of the solution of the corresponding homogeneous problem. The uniqueness of the latter is assured by lemma 1 .

Corollary: Equation (4) has a unique solution, even if the assumption that $\phi(P)$ be nonnegative is omitted.

Proof: Let $\phi$ be a negative lower bound of $\phi(\mathrm{P})$. Lemma (2) can be applied to the integral equations

$$
\begin{gathered}
\mathrm{u}_{1}(P)=\int_{G} u_{1}(Q) d F(Q, P)-\underline{\phi} \\
u_{2}(P)=\int_{G} u_{2}(Q) d F(Q, P)+\phi(P)-\phi
\end{gathered}
$$


$u(P)=u_{2}(P)-u_{1}(P)$ is then the solution of (4).

Lemma 3: If $\underline{\phi}$ and $\bar{\phi}$ are constants such that

$$
\underline{\phi} \leq \phi(P) \leq \bar{\phi} \text { in } G,
$$

and if $W(P)$ is the solution of

$$
W(P)=\int_{G} W(Q) d F(Q, P)+1,
$$

then the solution of (4) satisfies the inequalities

$$
\underline{\phi} W(P) \leq u(P) \leq \bar{\phi} W(P) .
$$

Proof: The function $\bar{u}(P)=\bar{\phi} W(P)$ is the solution of

$$
\bar{u}(P)=\int_{G} \bar{u}(Q) d F(Q, P)+\bar{\phi} .
$$

Subtracting (4) from this we see that $u(P)-u(P)$ is the solution of a similar integral equation with $\phi-\phi(P)$ as nonhomogeneous term. From lemma 2 it follows that $\bar{u}(P)-u(P)$ is nonnegative. The other inequality of (7) is similarly proved.

\section{The Integral Equatian for the Mean Duration}

Let $p_{n}(P)$ be the probability that the duration of a random walk starting at $P$ be exactly $n$. Because of the remark following the assumption $(A)$, we have

$$
\sum_{n=0}^{\infty} p_{n}(P)=1
$$

if we assume-as we shall-that assumption (A) is satisfied.

For a point outside $G$

$$
p_{n}(P)=\delta_{0 n}(P \text { not in } G)
$$

where $\delta_{0 n}$ is Kronecker's symbol. The expected duration of a random walk is - by definition-

$$
W(P)=\sum_{n=0}^{\infty} n p_{n}(P)
$$

if this series converges. Otherwise, the expected duration is infinite. We shall prove

Theorem 1. If assumption (A) is satisfied, $W(P)$ is finite and is the unique solution of the integral equation

$$
W(P)=\int_{G} W(Q) d F(Q, P)+1 .
$$

Proof: The probability $p_{n}(P)$ satisfies the relation

$$
p_{n+1}(P)=\int p_{n}(Q) d F(Q, P), n \geq 0 .
$$

Let

$$
W_{n}(P)=\sum_{\nu=0}^{n} \nu p_{\nu}(P)
$$

then we obtain from (10) the relation

$$
W_{n+1}(P)=\int W_{n}(Q) d F(Q, P)+\sum_{\nu=0}^{n+1} p_{\nu}(P)
$$

Reference to lemma 2 completes the proof.

If $F(Q, P)$ is a discrete distribution with a finite number of discontinuities, and if the position of these discontinuities is independent of $P$, then the integral equation (9) reduces to a difference equation. The simplest cases can be solved explicitly; see, e. g. [3, p. 286.]

\section{The Asymptotic Differential Equation}

In many applications the steps of the random walk are likely to be small. In order to describe more precisely the sense in which this statement is to be understood we assume, following Petrovsky, that the transition function depends on a small parameter $\mu$ in such a way that the following assumptions are satisfied.

Assumptions (B). Denote by $a_{i}(P, \mu), b_{i k}(P, \mu)$, $(i, k=1,2)$ the first and second moments of $F(Q, P)$ about the point $P$. Then

$$
\begin{aligned}
& a_{i}(P, \mu)=\alpha_{i}(P) \mu+o(\mu) \\
& b_{i k}(P, \mu)=\beta_{i k}(P) \mu+o(\mu) .
\end{aligned}
$$

The functions $o(\mu)$ may depend on $P$, but they are to have the indicated order uniformly in $G$. The $\alpha_{i}(P)$ and $\beta_{i k}(P)$ are assumed to be in class $C^{(2)}$ in $G+C$.

Assumption $(L)$. Let $K_{r}(P)$ denote the circle $P Q \leq r$, with center at $P$. Then

$$
\int_{E-K_{r}(P)} \overline{P Q}^{2} d F(Q, P)=o(\mu),
$$

here $o(\mu)$ depends on $P$ and $r$. The relation is to hold uniformly for $P$ in the closure of $G$, for any fixed $r>0$.

In the applications $\mu$ may measure the smallness of the mesh, if the walk takes place in a lattice, the smallness of the standard deviation, if the transition distribution is normal, the smallness of the time intervals between observations, if the walk is actually a motion in time, etc.

The definition of the $\beta_{i k}$ is readily seen to imply that $\beta_{11} \beta_{22}-\beta_{12} \geq 0$. We require, beyond this, Assumption $(\bar{E})$. In $G$ and on its boundary,

$$
\beta_{11} \beta_{22}-\beta_{12}^{2}>0 \text {. }
$$

This assumption will enable us to make use of the existence theorems in the theory of elliptic differential equations. 
Finally, we have to require a certain degree of smoothness of the boundary $C$ of $G$. In particular, our results remain valid for polygonal domains.

Assumption $(S) . \quad C$ has a continuously turning tangent.

This restriction can be weakened. The necessary arguments can be found in [4, p. 438].

We shall show that the function $w=\mu W$ remains finite, as $\mu \rightarrow 0$ and converges to the solution of the differential problem

$$
\begin{gathered}
\mathscr{L}[V]+1=0 \text { in } G \\
V=0 \text { on } C
\end{gathered}
$$

where,

$$
\mathscr{L}[V]=\frac{1}{2} \beta_{11} V_{x x}+\beta_{12} V_{x y}+\frac{1}{2} \beta_{22} V_{y y}+\alpha_{1} V_{x}+\alpha_{2} V_{y} .
$$

Because of assumption (E) the differential problem (11) is elliptic and possesses in $G$ a unique solution.

The basic idea of the proof is to show that $V$ satisfies an integral equation little different from the one for $W$. In order to avoid extraneous difficulties near the boundary $C$ it is convenient to replace $V(P)$ by a slightly different function $V_{\delta}(P)$ defined in a larger domain $G^{\prime \prime}$. This can be done by constructing a twice continuously differentiable mapping

$$
x^{\prime}=f(x, y ; \delta), y^{\prime}=g(x, y ; \delta),(x, y \text { in } G)
$$

which is continuous in $\delta$, for $\delta \geq 0$, together with its first and second derivatives with respect to $x$ and $y$, and has the following properties: (a) It reduces to the identity for $\delta \rightarrow 0$. (b) It is, for all $\delta$, the identity transformation in a subdomain $G^{\prime}$ of $G$ that tends to $G$ as $\delta \rightarrow 0$. (c) It maps $G$ into a domain $G^{\prime \prime}$ containing $G$ in its interior for $\delta>0$.

For the explicit construction of such a mapping with the help of assumption (S) we refer to [4].

If we define $V_{\delta}(P)$ in $G$ by

$$
V_{\delta}\left(x^{\prime}, y^{\prime}\right)=V(x, y),(x, y \text { in } G)
$$

then this new function is defined and twice continuously differentiable in $G^{\prime \prime}$. It tends to $V(P)$, uniformly in $G$ together with its first and second derivatives. We extend the definition into the whole plane $E$ by setting

$$
V_{\delta}(P)=0 \text { in } E-G^{\prime \prime} .
$$

In order to estimate the integral

$$
J=\int V_{\delta}(Q) d F(Q, P)
$$

for $P$ in $G$ we denote by $K(P, \delta)$ a circle about $P$ with radius $r(\delta)$ depending on $\delta$ but not on $P$, such that $K(P, \delta)$ is in $G^{\prime \prime}$ whenever $P$ is in $G$. We define

$$
J_{1}=\int_{K(P, \delta)} V_{\delta} d F, J_{2}=\int_{E-K(P, \delta)} V_{\delta} d F,
$$

then $J=J_{1}+J_{2}$.

Now if $A$ is an upper bound of $\left|V_{\delta}\right|$, for all $\delta$ with $0 \leq \delta \leq \delta_{0}$

$$
\left|J_{2}\right| \leq \frac{A}{r^{2}(\delta)} \int_{E-K(P, \delta)} \overline{P Q}^{2} d F(Q, P) .
$$

Hence, by assumption $(\mathrm{L})$,

$$
J_{2}=o(\mu), \quad \text { for fixed } \delta .
$$

For the calculation of $J_{1}$ we use Taylor's formula, for $P$ in $G, Q$ in $K(P, \delta)$, setting

$$
V_{\delta}(Q)=V_{\delta}(P)+M\left[V_{\delta}(P)\right]+Q\left[V_{\delta}(P)\right]+R\left[V_{\delta}\right],
$$

where

$$
\begin{aligned}
& M[V]=(\xi-x) V_{x}(P)+(\eta-y) V_{y}(P) \\
Q[V]= & \frac{1}{2}\left\{(\xi-x)^{2} V_{x x}(P)+2(\xi-x)(\eta-y) V_{x y}(P)+\right. \\
& \left.(\eta-y)^{2} V_{\eta \eta}(P)\right\}
\end{aligned}
$$

and

$$
R[V]=Q[V(\widetilde{P})]-Q[V(P)], \widetilde{P} \text { in } K(P, \delta)
$$

i. e.,

$$
R\left[V_{\delta}\right]=\overline{P Q}^{2} \rho(P, Q, \delta)
$$

with

$\lim _{\delta \rightarrow 0} \rho(P, Q, \delta)=0$ uniformly for $P$ in $G, Q$ in $K(P, \delta)$.

In these formulas $x, y$ and $\xi, \eta$ are the coordinates of $P$ and $Q$, respectively. We now integrate (14) termwise with respect to $Q$ over $K(P, \delta)$ and discuss the terms separately. We have, e.g., by assumption (B),

$\int_{K(P, \delta)}(\xi-x) d F=\mu \alpha_{1}+o(\mu)-\int_{E-K(P, \delta)}(\xi-x) d F$,

but

$$
\left|\int_{E-K(P, \delta)}(\xi-x) d F\right| \leq \frac{1}{r(\delta)} o(\mu)
$$

by assumption $(\mathrm{L})$, and hence

$$
\int_{K(P, \delta)}(\xi-x) d F=\mu \alpha_{1}+o(\mu), \text { for fixed } \delta .
$$

Similar arguments apply to 


$$
\int_{K(P, \delta)}^{d} d F, \quad \int_{K(P, \delta)}(\eta-y) d F, \text { etc. }
$$

Also,

$$
\int_{K(P, \delta)} R\left[V_{\delta}\right] d F=\rho_{1}(P, \delta) 0(\mu)
$$

by virtue of assumption (B). Here $\rho_{1}(P, \delta)$ tends to zero, uniformly in $G$, as $\delta \rightarrow 0$, because of (1.6), and $0(\mu) / \mu$ remains bounded as $\delta \rightarrow 0$. Formulas $(1.3),(1.7),(1.8)$ and the formulas for $\int_{K(P, \delta)} d F$, $\int_{K(P, \delta)}(\eta-y) d F$, etc., analogous to $(1.7)$, when applied to (1.4), yield

$$
J=V_{\delta}(P)+\mu L\left[V_{\delta}\right]+\rho_{1}(P, \delta) 0(\mu)+o(\mu) .
$$

Now let $\epsilon>0$ be assigned arbitrarily (but less than $A)$, and choose a $\delta,\left(0<\delta \leq \delta_{0}\right)$, so small that the following inequalities hold:

$$
\left|L\left[V_{\delta}\right]+1\right| \leq \epsilon / 24 A \text { in } G
$$

This is possible because of (11) and the properties of $V_{\delta}$ as described above.

$$
\left|\rho_{1}(P, \delta) 0(\mu)\right|<\mu \epsilon / 24 A
$$

Here the remark after formula (18) is used.

$$
\begin{aligned}
& \left|V_{\delta}-V\right| \leq \frac{\epsilon}{4} \text { in } G \\
& \left|V_{\delta}\right| \leq \frac{\epsilon}{4} \text { in } G^{\prime \prime}-G
\end{aligned}
$$

This last inequality is the only point where the boundary condition in (11) is needed.

After $\delta$ has been fixed in this fashion we choose $\mu_{0}>0$ so small that the $o(\mu)$ in (19) satisfies the inequality

$$
|\mathrm{o}(\mu)| \leq \mu \epsilon / 24 A \text { in } G, \text { for }|\mu| \leq \mu_{0} .
$$

From (20), (21) and (24) it follows that (19) can be written

$$
\int V_{\delta}(Q) d F(Q, P)=V_{\delta}(P)-\mu g(P, \mu),
$$

where

$$
|g(P, \mu)-1| \leq \epsilon / 8 A \text { for } P \text { in } G,|\mu| \leq \mu_{0} .
$$
into

For the comparison of $V_{\delta}$ with $\mu W$ we split $V_{\delta}$ where

$$
V_{\delta}=V_{\delta}^{(1)}+V_{\delta}^{(2)},
$$

$$
\int_{G} V_{\delta}^{(1)} d F=V_{\delta}^{(1)}-\mu g, V_{\delta}^{(1)}=0 \text { in } E-G
$$

$$
\int V_{\delta}^{(2)} d F=V_{\delta}^{(2)} \text { in } G, V_{\delta}^{(2)}=V_{\delta} \text { in } E-G .
$$

The integral equations (27) and (28) have unique solutions, by the corollary to lemma 2, and by lemma 1, respectively. If we apply lemma 3 to $(27)$, it follows from (9) and (26) that

$$
\mu\left(1-\frac{\epsilon}{8 A}\right) W \leq V_{\delta}^{(1)} \leq \mu\left(1+\frac{\epsilon}{8 A}\right) W .
$$

Next we apply the corollary of lemma 1 to (28) and use the inequality (23). This shows that

$$
\left|V_{\delta}^{(2)}\right| \leq \frac{\epsilon}{4}
$$

The inequalities (29) imply

$$
\left|\mu W-V_{\delta}^{(1)}\right| \leq \frac{\epsilon}{2}
$$

In fact, we have, for $\epsilon \leq 4 \mathrm{~A}$,

$$
\frac{1}{1-\epsilon / 8 A} \leq 1+\frac{\epsilon}{4 A}
$$

and, therefore, from the first inequality in (29),

$$
\mu W-V_{\delta}^{(1)} \leq \frac{\epsilon}{4 A} V_{\delta}^{(1)} .
$$

By the definition of $V_{\delta}^{(1)}$ and inequality (30),

$$
\left|V_{\delta}^{(1)}\right| \leq 2 A \text {. }
$$

If this is combined with inequality (32), one of the two inequalities equivalent to (31) is at hand. The second inequality is proved similarly.

Finally, adding inequality (22) to (31) the desired formula

$$
|\mu W-V| \leq \epsilon, \text { for }|\mu| \leq \mu_{0}
$$

is proved.

We summarize this result as a theorem.

Theorem 2. If assumptions (A), (B), (L), (E), and $(\mathrm{S})$ are satisfied, then the mean duration $W(P)$ of the random walk satisfies the asymptotic relation

$$
\lim _{\mu \rightarrow 0} \mu W(P)=V(P),
$$

uniformly in $G$, where $V(P)$ is the solution of the elliptic boundary value problem (11).

\section{Bounds for the Mean Duration in a Simple Case}

From the viewpoint of numerical application the preceding theory is of interest mostly as a basis for 
finding reasonable bounds for the expected duration There exists several methods for this. But since this investigation was originally motivated by questions connected with the "Monte Carlo" technique of solving differential equations experimentally by means of random walks, we shall disregard methods which amount themselves essentially to an approximate solution of such a differential equation. A general procedure for obtaining bounds for $W(P)$ is given in [2]. Here we shall limit ourselves to the special case that $L[u]$ is a constant multiple of Laplace's operator. Then it is possible to obtain much more precise information, even if the space is again assumed to be $n$-dimensional.

In other words, we require that

$$
\beta_{i K}(P)=k \cdot \delta_{i K}, \alpha_{i}(P)=0, \quad i, K=1,2, \cdots, n
$$

The differential problem (11) now assumes the form

$$
\Delta V+\frac{2}{k}=0 \text { in } G, V=0 \text { on } C
$$

where $\Delta$ is Laplace's operator in $n$ dimensions.

The constant $k$ is related to the mean square of the step length, $s^{2}=\sum_{i=1}^{n} b_{i i}(P)$ by the formula

$$
s^{2}=n \mu k+o(\mu)
$$

There is therefore a $\mu_{1}>0$ such that for $|\mu| \leq \mu_{1}$ it is possible to use $s^{2}$ itself as a parameter $\mu$. Without loss of generality we can therefore set

$$
\mu=S^{2}
$$

and, hence,

$$
k=\frac{1}{n},
$$

and

$$
\Delta V+2 n=0 \text { in } G, V=0 \text { on } C
$$

For those arguments below, where we let $n$ approach infinity we need the further assumption that $\mu_{1}$ can be chosen independently of $n$. Random walks of the type described above will be called symmetric.

The two most interesting special cases of the type considered are

(a) The random walk takes place in an orthogonal net of mesh length $h$ with transition probability $1 / 2 n$ in each of the $2 n$ possible directions.

Here $a_{i}=0, b_{i K}=h^{2} \delta_{i K} / n$.

Assumptions (B), (L), and (E) are satisfied, if $\mu$ is defined by

$$
\mu=h^{2} \text {. }
$$

Then (35) and (34) are satisfied.

(b) The transition probability is normal with

$$
a_{i}=0, b_{i K}=\sigma^{2} \delta_{i K} .
$$

If we define

$$
\mu=n \sigma^{2}
$$

formulas (34) and (35) remain again valid.

Theorem 3. If the conditions (33) are satisfied the mean duration of a random walk in an $n$-dimensional sphere of radius a satisfies the asymptotic formula

$$
W(P)=\frac{a^{2}-r^{2}}{s^{2}}[1+\epsilon(s)]
$$

where $r$ is the distance of $P$ from the center, $s^{2}$ the mean square of the step length, and $\lim \epsilon(s)=0$. The most interesting aspect of this formula is its asymptotic independence of the dimension.

Proof: If $G$ is a sphere in $n$ dimensions, the solution of problem (36) is $V=a^{2}-r^{2}$. Theorem 3 is therefore an immediate consequence of theorem 2 and formula (34).

Corollary 1: Let $v$ be the volume of the $n$-dimensional sphere of radius $a$. Then the value of $W(P)$ at the center 0 of the sphere is

$$
W(0)=\Gamma^{2 / n}\left(\frac{n+2}{2}\right) \pi^{-1} s^{-2} v^{2 / n}[1+\epsilon(s)] .
$$

Corollary 2:

$$
\lim _{n \rightarrow \infty} W(0) / n=A(s), \text { where } A(s) \neq 0
$$

This follows by a simple application of Sterling's formula to the right side of the preceding formula.

If $G$ is not a sphere, the equality (40) can be replaced by an inequality, if we make use of the following lemma.

Lemma 4. Let $P$ be a fixed point in $n$-dimensional space and denote by $\{B\}$ the set of all $n$-dimensional domains of constant volume $v$ containing $P$ in its interior and having a boundary which is sectionally in Class $C^{(2)}$. Let $u_{B}(P)$ be the value at $P$ of the solution of the differential problem

$$
\Delta u+1=0 \text { in } G, u=0 \text { on } C .
$$

Then $u_{B}(P)$ assumes its maximum with respect to the Class $\{B\}$, if $B$ is the sphere $K$ with center at $P$.

Proof: Let $r$ be the distance $\overline{P Q}$ and denote by $G_{B}(Q)$ Green's function in $B$, if one argument point is fixed at $P$. For the sphere $K$ of radius $a$ about $P$ Green's function is of the form

$$
G_{K}(Q)=c_{1}(n)\left[r^{2-n}-a^{2-n}\right], n>2
$$

where $c_{1}(n)$ is a constant depending on the dimension $n$. For any domain $B$ we have 


$$
u_{B}(P)=\int_{B} G_{B}(Q) d Q
$$

Denote by $v_{B}(\mathrm{P}), \mathrm{P} \geq 0$ the volume of the "level" surface $G_{B}(Q)=\rho$. For the sphere $K$ we have, from $(42)$,

$$
v_{K}(\rho)=c_{2}(n)\left(\rho+a^{2-n}\right)^{n /(2-n)}
$$

We now make use of the inequality

$$
v_{B}(\rho) \leq v_{K}(\rho) \text {, for all } B \text { of }\{B\}
$$

which is an easy consequence of the classical extremal property of the capacity of a spherical shell. (cf. Szegö [6], in particular p. 588. Szegö's proof for the three-dimensional case extends readily to higher dimensions.) From (43) we have

$$
u_{B}(P)=-\int_{0}^{\infty} \rho d v_{B}(\rho)=-\left[\rho v_{B}(\rho)\right]_{0}^{\infty} \int_{0}^{\infty} v_{B}(\rho) d \rho .
$$

The first term in the right member is zero, for $v_{B}(0)=v$ and $\lim \rho v_{B}(\rho)=0$, since $\rho v_{K}(\rho)$ - which by $(45)$ at least equals $\rho v_{B}(\rho)$-tends to zero, as $\rho \rightarrow \infty$, in consequence of (44). A reference to (45) now completes the proof of the lemma for $n>2$. The modifications for $n=2$ are trivial.

From this lemma and corollary 1 of theorem 3 we obtain immediately the following result.

Theorem 4: The mean duration of a symmetric random walk in an $n$-dimensional domain of volume $v$ satisfies the inequality

$$
W(P) \leq \pi^{-1} \Gamma^{2 / n}\left(\frac{n+2}{2}\right) s^{-n} v^{2 / n}[1+\epsilon(s)] .
$$

Corollary 2 to theorem 3 implies that the right member of inequality (46) tends to infinity with order $n$, as $n \rightarrow \infty$, provided the volume is kept constant. But an explicit calculation shows that the increase, for small integers $n$ is slower. In fact, if we write the right member of inequality (46) in the form

$$
c(n) v^{2 / n} s^{-2}[1+\epsilon(s)]
$$

we have

\begin{tabular}{|c|c|c|c|c|c|c|c|}
\hline$n$ & 1 & 2 & 3 & 4 & 5 & 6 & 7 \\
\hline \multicolumn{1}{c|}{$c(n)$} & .250 & .318 & .385 & .450 & .515 & .578 & .642 \\
\hline
\end{tabular}

\section{VI, Random Walks in an $n$-dimensional Cube}

The $n$-dimensional cube is another domain for which problem (36) can be easily solved. It seems intuitively plausible that the value of $W(P)$ at the center of such a cube will have the same order of magnitude in $n$ as the value for the sphere, given by (41). This is, however, not the case. We shall, in fact, prove the following theorem.

Theorem 5: For a symmetric random walk problem let $W_{n}^{s}$ denote the mean duration of walks starting at the center of an $n$-dimensional sphere of volume $v$, and denote by $W_{n}^{c}$ similarly the mean duration for a walk starting at the center of an $n$-dimensional cube of the same volume $v$. Then

$$
\lim _{n \rightarrow \infty} W_{n}^{c} / W_{n}^{s}=0
$$

but

$$
\lim _{n \rightarrow \infty} n^{\epsilon} W_{n}^{c} / W_{n}^{s}=\infty,(\epsilon>0 \text {, arbitrary }) .
$$

Proof: It suffices to consider the unit cube $B$, defined by $\left|x_{i}\right|<\frac{1}{2},(i=1, \cdots, n)$. The eigenfunctions of $\Delta u$ in $B$ are

$u_{K_{1}} \cdots K_{n}\left(x_{1}, \cdots, x_{n}\right)=\prod_{i=1}^{n} \cos K_{i} \pi x_{i}, K_{i}>0, K_{i}$ odd, and the corresponding eigenvalues are

$$
\lambda_{K_{1}} \cdots K_{n}=\pi^{2} \sum_{i=1}^{n} K_{i}^{2} .
$$

These eigenfunctions are orthogonal. Their norm is $2^{-n}$, since

$$
\int_{-\frac{1}{2}}^{\frac{1}{2}} \cdots \int_{-\frac{1}{2}}^{\frac{1}{2}} \prod_{i=1}^{n} \cos ^{2} K_{i} \pi x_{i} d x_{1} \cdots d x_{n}=2^{-n} .
$$

It follows that the multiple Fourier coefficients $c_{K_{1}} \cdots K_{n}$ of the representation

$1=\sum_{K_{1} \ldots K_{n}} c_{K_{1} \ldots K_{n}} u_{K_{1} \ldots K_{n}}\left(x_{1} \ldots x_{n}\right),\left(\right.$ odd $\left.K_{1} \ldots K_{n}\right)$ valid in $B$, are

$$
c_{K_{1}} \ldots K_{n}=\left(\frac{4}{\pi}\right)^{n}(-1)^{\left(K_{i}-1\right) / 2} / \prod_{i=1}^{n} K_{i} .
$$

If we now substitute for $u$ in $\Delta u+1=0$ a Fourier series

$$
\begin{aligned}
u= & \sum_{K_{1}} \ldots K_{n} a_{K_{1}} \ldots K_{n} u_{K_{1}} \ldots K_{n}\left(x_{1}, \ldots, x_{n}\right), \\
& \left(\text { odd } K_{1}, \ldots, K_{n}\right)
\end{aligned}
$$

comparison of coefficients shows that

$$
\lambda_{K_{1} \ldots K_{n}} a_{K_{1} \ldots K_{n}}=c_{K_{1}} \ldots K_{n}
$$

that is, the value of $u$ at the center $x_{i}=0$ of the cube is

$$
\begin{aligned}
u(0)= & \pi^{-2}\left(\frac{4}{\pi}\right)^{n} \sum_{K_{1}}, \ldots K_{n} \prod_{i=1}^{n}(-1)^{\left(K_{i}-1\right) / 2} / \prod_{i=1}^{n} K_{i} \sum_{i=1} K_{i}^{2} \\
& \left(\operatorname{odd} K_{1}, \ldots, K_{n}\right)
\end{aligned}
$$


This $n$-fold sum can be simplified by means of the substitution

$$
\left(\sum_{i=1}^{n} K_{i}^{2}\right)^{-1}=\int_{0}^{\infty} e^{-x \sum_{i=1}^{n} K_{i}^{2}} d x
$$

followed by an interchange of summation and integration, which is not difficult to justify (see, e. g. [1] I, p. 331). Then we obtain

$$
\begin{aligned}
u(0)= & \pi^{-2}\left(\frac{4}{\pi}\right)^{n} \int_{0}^{\infty} \sum_{K_{1}} \ldots K_{n} \prod_{i=1}^{n} \frac{(-1)^{\left(K_{i}-1\right) / 2}}{K_{i}} e^{-x K_{i}^{2}} d x, \\
& \left(K_{1}, \ldots, K_{n} \text { odd }\right)
\end{aligned}
$$

that is

$$
u(0)=\pi^{-2}\left(\frac{4}{\pi}\right)^{n} \int_{0}^{\infty}\left\{\sum_{j=0}^{\infty}(-1)^{j} \frac{e^{-(2 j+1)^{2} x}}{2 j+1}\right\}^{n} d x .
$$

In order to investigate the asymptotic dependence of $u(0)$ on $n$ we make use of the formula

$$
\sum_{m=-\infty}^{\infty} e^{-x(m+T)^{2}}=\sqrt{\frac{\pi}{x}} \sum_{n=-\infty}^{\infty} \exp \left\{-x T^{2}+(x T+\pi i n)^{2} / x\right\}
$$

(see, e.g. [5], p. 32), which is essentially the reciprocity formula for the $\theta$-function. If we make the substitution $T=i y / x$ and integrate with respect to $y$ between 0 and $\pi / 4$ we obtain from this formula the series

$$
f(x)=-\frac{\pi}{2}+\sqrt{\frac{\pi}{x}} \sum_{x=-\infty}^{\infty} \int_{0}^{\pi / 4} \exp \left[-(y+\pi n)^{2} / x\right] d y
$$

for the function

$$
f(x)=\sum_{j=0}^{\infty}(-1)^{j} \frac{e^{-(2 j+1)^{2} x}}{2 j+1} .
$$

Setting $t=(y+\pi n / \sqrt{x}$ in the integrals above, this becomes

$f(x)=-\frac{\pi}{2}+\sqrt{\pi}\left\{\int_{0}^{T / 4 \sqrt{x}} e^{-t^{2}} d t+\sum_{n=1}^{\infty} \int_{\pi\left(n-\frac{1}{4}\right) / \sqrt{x}}^{\pi\left(n+\frac{1}{4}\right) / \sqrt{x}} e^{-t^{2}} d t\right\}$

From (48) we conclude

$$
f(x)<-\frac{\pi}{2}+\sqrt{\pi} \int_{0}^{\infty} e^{-t^{2}} d t=\frac{\pi}{4}, \text { for } x>0
$$

and from the definition of $f(x)$,

Hence,

$$
f(x)<e^{-x}
$$

$$
\begin{aligned}
u(0) & =\pi^{-2}\left(\frac{4}{\pi}\right)^{n}\left\{\int_{0}^{\epsilon}+\int_{\epsilon}^{1}+\int_{1}^{\infty} f^{n}(x) d x\right\} \\
& \leq \pi^{-2}\left\{\epsilon+\left(\frac{4}{\pi} c_{1}\right)^{n}(1-\epsilon)+\left(\frac{4}{\pi} c_{2}\right)^{n}\right\}
\end{aligned}
$$

where $\epsilon$ is an arbitrarily small positive number and $c_{1}$ and $c_{2}$ are constants less than $\pi / 4$. Therefore

$$
\lim _{n \rightarrow \infty} u(0)=0 \text {. }
$$

The first part of theorem 5 is now an immediate consequence of theorem 2 and formulas (36) and (41).

In order to estimate the rate of convergence of $u(0)$ from below, we observe that

$$
u(0) \geq \pi^{-2}\left(\frac{4}{\pi}\right)^{n} \int_{0}^{1} f^{n}(x) d x .
$$

In the right member we use the inequality

$$
f(x) \geq-\frac{\pi}{2}+\sqrt{\pi} \int_{0}^{\infty} e^{-t^{2}} d t-\sqrt{\pi} \int_{\pi / 4 \sqrt{x}}^{\infty} e^{-t^{2}} d t,
$$

which follows from (48), combining it with the inequality

$$
\int_{z}^{\infty} e^{-t^{2}} d t<\frac{1}{2 z} e^{-z^{2}}, \text { for } z>0,
$$

which is readily proved by an integration by parts. Then we obtain

$$
f(x) \geq \frac{\pi}{4}\left(1-\frac{8 \sqrt{x}}{\pi^{3 / 2}} e^{-\pi^{2} / 16 x}\right) \geq \frac{\pi}{4}\left(1-2 x^{N}\right) \geq \frac{\pi}{4} e^{-2 x^{N}}
$$

for $0 \leq x \leq 1 ; N>0$, arbitrary. Inserting this in (49), we have, finally,

$$
\begin{aligned}
u(0) & \geq \pi^{-2} \int_{0}^{1} \exp \left[-2 n x^{k}\right] d x=\frac{\pi^{-2}}{k n^{1 / N}} \int_{0}^{n} e^{-2 \tau} \tau^{1 / N-1} d \tau \\
& \geq \text { const. } n^{1 / N} .
\end{aligned}
$$

This, in combination with formulas (36), (41), and theorem 2, proves the second part of theorem 5.

\section{Random Walks in $n$-Dimensional Ellipsoids}

In this section we collect some results concerning the mean duration of random walks in $n$-dimensional ellipsoids.

The "oblongness" of such an ellipsoid can be defined as the ratio of the longest to the shortest axis. The mean duration of a random walk of the class considered in the last two sections will be less for a walk starting from the center of such an ellipsoid than it would be for a sphere of same volume. The ratio between these two quantities will give an idea of the deterioration of the estimate (46) for oblong convex domains. For certain domains it may even be advantageous to estimate the mean duration of the random walk by its value in a circumscribed ellipsoid rather than by formula (46). We shall make use of a lemma concerning the arithmetic and geometric means of $n$ numbers which may be of some interest in itself. 
Theorem 6. The mean duration of a symmetric random walk in an $n$-dimensional ellipsoid with semiaxes $a_{1}, \ldots, a_{n}$ is

$$
W_{E}(P)=s^{-2}[1+\epsilon(s)] n\left(1-\sum_{i=1}^{n} \frac{x_{i}^{2}}{a_{i}^{2}}\right) / \sum_{i=1}^{n} \frac{1}{a_{i}^{2}}
$$

Proof. It is readily verified that the solution of problem (36) in such an ellipsoid is

$$
V(P)=n\left(1-\sum_{i=1}^{n} \frac{x_{i}^{2}}{a_{i}^{2}}\right) / \sum_{i=1}^{n} \frac{1}{a_{i}^{2}} .
$$

This fact and theorem 2 prove formula $(50)$.

Theorem $\%$. If $W_{S}(P)$ is the mean duration of a symmetric random walk for a sphere having the same volume as the ellipse, then

$$
W_{E}(0) / W_{S}(0)=[1+\epsilon(s)]\left\{\prod_{j=1}^{n} a_{j}^{-2}\right\}^{1 / n} / n^{-1} \sum_{j=1}^{n} a_{j}^{-2} .
$$

Proof: The volume of the ellipsoid is

$$
v=\pi^{n / 2} \Gamma^{-1}\left(n+\frac{1}{2}\right) \prod_{j=1}^{n} a_{j} .
$$

If we divide formula (50) for $P=0$ by the formula in corollary 1 to therorem 3 , and eliminate $v$ by means of formula (52), we obtain formula (51).

From now on we shall assume that the oblongness of the ellipsoid is prescribed. In view of the remarks at the beginning of this section it is of interest to investigate the minimum of the right member in (51) under this restriction. To this end we prove the following lemma:

Lemma 5: Let $t_{j}(j=1, \ldots ., n)$ be $n$ positive numbers such that the ratio of the largest to the smallest has a prescribed value $\rho$. Then the quotient of the geometric and the arithmetic mean of these numbers will be smallest when

$$
t_{1}=t_{2}=\cdots=t_{r} ; \quad t_{r+1}=t_{r+2}=\cdots=t_{n},
$$

where $r$ is an integer that satisfies the inequalities

with

$$
\chi(\rho) n \leq r \leq \chi(\rho) n+1,
$$

$$
\chi(\rho)=\frac{\rho}{\rho-1}-\frac{1}{\log \rho} .
$$

Proof: Without loss of generality we can assume

$$
t_{1}=1, t_{1} \leq t_{2} \leq \ldots . \leq t_{n}, t_{n}=\rho .
$$

We have to minimize the quantity

$$
F\left(t_{2}, \ldots, t_{n-1}\right)=\prod_{j=1}^{n} t_{j} /\left(\Sigma t_{j}\right)^{n}
$$

under the side conditions (53). As a function of $t_{s}$ alone, $(1<s<n), F$ has its only stationary value when

$$
\sum_{j=1}^{n} t_{j}-n t_{s}=0
$$

i.e.,

$$
t_{s}=\frac{1}{n-1}\left\{\sum_{j=1}^{n} t_{j}-t_{s}\right\}
$$

This stationary value is a maximum, since $F$ is zero when $t_{s}=0$ or $t_{s}=\infty$. Hence, as a function of $t_{s}$ alone, $F$ has no relative minimum and it assumes therefore its minimum for given values of $t_{j}(j+s)$ either when $t_{s}=t_{s-1}$ or when $t_{s}=t_{s+1}$. It follows that at the minimum of $F$, the $t_{j}$ consist of two groups such that

$$
t_{1}=t_{2}=\cdots=t_{r}, t_{r+1}=\cdots=t_{n-1}=t_{n}, 1 \leq r \leq n-1
$$

the minimum will therefore be among the values of

$$
\psi(r)=\frac{\rho^{n-r}}{[r+(n-r) \rho]^{n}}, 1 \leq r \leq n-1 .
$$

We find that $\psi(r)$ has exactly one stationary value, which occurs at

$$
r=r_{0}=n \chi(\rho)=n\left(\frac{\rho}{\rho-1}-\frac{1}{\log \rho}\right) .
$$

This stationary value is a minimum, for $\psi(r)$ is continuously differentiable and positive in the interval $-\infty<r<n \rho /(\rho-1)$, it is infinite at $r=-\infty$ and $r=$ $n \rho /(\rho-1)$, and $r_{0}$ lies in this interval. Moreover, $\lim _{\rho \rightarrow 1} \chi(\rho)=\frac{1}{2}, \chi(\rho)$ increases with $\rho$, and $\chi(\infty)=1$. $\stackrel{\rho \rightarrow 1}{\text { Hence }}$

$$
\frac{n}{2}<r_{0}<n \text {. }
$$

If $r_{0}$ is an integer, this is the admissible value of $r$ for which $\psi(r)$ assumes its minimum. Otherwise, it is one of the two neighboring integers. This completes the proof of the lemma.

Corollary 1: For sufficiently small $\rho>1$ the integer $r$ of lemma 5 differs by less than unity from $n / 2$. As $\rho$ increases, so does $r$. For sufficiently large $\rho$ we have $r=n-1$. Also, $r$ is never less than $n / 2$.

Corollary 2: For $n=3$ we have always $r=2$.

Proof: One shows easily that, for $\rho>1$,

$$
\psi(1) / \psi(2)>1 \text {. }
$$

Theorem 8: With the notations and assumption of theorem 7 denote by $m(\omega)$ the minimum of the ratio $W_{E}(0) / W_{S}(0)$ for all ellipsoids of given oblongness $\omega$.

Then

$$
\begin{aligned}
& \lim _{n \rightarrow \infty}\{m(\omega) \cdot[1+\epsilon(s)]\}= \\
& {\left[\chi(\sqrt{\omega}) \omega^{2(1-\chi(\sqrt{\omega}))}+\omega^{2}(1-\chi(\sqrt{\omega}))\right]^{-1} .}
\end{aligned}
$$


Proof: Without loss of generality we set

hence

$$
a_{1} \geq a_{2} \geq \ldots \geq a_{n}
$$

$$
\omega=\frac{a_{1}}{a_{n}}
$$

We apply lemma 5 to formula (51) with

$$
\begin{aligned}
& t_{j}=a_{j}^{-2}, \text { i. e., } \\
& \omega=\left(\frac{t_{n}}{t_{1}}\right)=\rho^{2} .
\end{aligned}
$$

The minimum of the right side of (51) is then

$$
\begin{aligned}
& {[1+\epsilon(a)] n a_{1}^{-2 r / n} a_{n}^{-2(n-r) / n} / r a_{1}^{-2}+(n-r) a_{n}^{-2}=} \\
& \frac{\omega^{-2 r / n}}{\frac{r}{n} \omega^{-2}+1-\frac{r}{n}}[1+\epsilon(s)] .
\end{aligned}
$$

As $n \rightarrow \infty, r / n \rightarrow \chi(\rho)=\chi(\sqrt{\omega})$, and formula (55) is at hand. Theorem 8 shows that for sufficiently large $n$ and $\omega>1$

$$
m(\omega)<\omega^{2(x(\sqrt{\omega})-1)}<1
$$

On the other hand, $m(\omega)$ does not tend to zero as $n \rightarrow \infty$. For large $n$, random walks starting from the center of any ellipsoid of prescribed oblongness are on the average longer than those in $n$-dimensional cube of same volume. If $r$ is kept constant for all $n$ in (56), it represents the ratio $W_{e}(0) / W_{s}(0)$ for ellipsoids of given oblongness $r$ of whose axes have maximal length while the remaining $n-r$ have minimal length. For this type of ellipsoids $W_{E}(0) / W_{S}(0)$ tends to 1 as $n \rightarrow \infty$. The corresponding random walks are, therefore, on the average longer, for large $n$, than for ellipsoids of the minimal type.

\section{References}

[1] R. Courant and D. Hilbert, Methoden der mathematischen Physik 1, second ed., (J. Springer, Berlin, 1931).

[2] J. H. Curtiss, Sampling methods applied to differential and difference equations. To appear in the Proceedings of a Scientific Computation Forum, held by the International Business Machines Corporation, Endicott, N. Y., November 1949.

[3] W. Feller, An introduction to probability theory and its applications (J. Wiley \& Sons, Inc., New York, N. Y., 1950).

[4] I. Petrowsky, Über das Irrfahrtproblem, Math. Ann. 109, 425-444 (1934).

[5] J. B. Rosser, Theory and application of $\int_{0}^{z} e^{-x^{2}} d x$ and $\int_{0}^{z} e^{-p y^{2}} d y \int_{0}^{y} e^{-x^{2}} d x$, part I (Mapleton House, Brooklyn, N. Y., 1948).

[6] Szegö, Über einige Extremalaufgaben der Potentialtheory. Math. Z. 31, 583-593 (Feb. 1930).

Los Angeles, August 21, 1950. 